\title{
Radial evolution of intermittent heat flux in solar coronal holes
}

\author{
S. A. Markovskii and J. V. Hollweg \\ Space Science Center, University of New Hampshire, Durham, NH 03824, USA \\ Received: 8 September 2004 - Revised: 20 October 2004 - Accepted: 1 November 2004 - Published: 8 November 2004 \\ Part of Special Issue "Advances in space environment turbulence"
}

\begin{abstract}
Recently, we suggested that the source of ion heating in solar coronal holes is small-scale reconnection events (microflares) at the coronal base. The microflares launch intermittent heat flux up into the corona exciting ion cyclotron waves through a plasma microinstability. The ions are heated by these waves during the microflare bursts and then evolve with no energy input between the bursts. The overall coronal heating by this mechanism is a summed effect of all microflare bursts during the expansion time of the solar wind and adiabatic cooling between the microflares. The intermittent heat flux produced by the microflares was modeled as electron beams with constant speed and temperature for simplicity. In this paper, we consider a more sophisticated model of the heat flux taking into account the action of the mirror force and the charge separation electric field on the beam particles. We show that the radial evolution of the heat flux is determined mainly by the beam expansion along the magnetic field roughly at the root mean square velocity of the beam particles, while the variation of the beam bulk speed and thermal energy is less important.
\end{abstract}

\section{Introduction}

A possible source of heating of the solar corona is turbulent fluctuations in the ion cyclotron frequency range. This hypothesis is strongly supported by the observational data from the UVCS instrument on the SOHO spacecraft. In particular, the data show that the heating increases the ion temperature mostly in the direction perpendicular to the background magnetic field (e.g. Kohl et al., 1998; Dodero et al., 1998; Antonucci et al., 2000) and different ions are heated with different efficiency (e.g. Kohl et al., 1998, 1999). All these and other properties can be explained by the ion cyclotron wave-particle interaction (e.g. Hollweg and Isenberg, 2002). It should be mentioned that other possibilities have been dis-

Correspondence to: S. A. Markovskii

(sergei.markovskii@unh.edu) cussed too; see Hollweg and Isenberg (2002) for a review. These possibilities cannot be ruled out because there is no direct evidence for the ion cyclotron waves, but the indirect evidence is rather convincing and we will assume that the ion cyclotron waves do exist in the corona.

Then, the next question to ask is, where do these waves come from and how are they replenished to provide the energy source for the corona and the solar wind. This aspect of the coronal heating is much less understood. Several mechanisms of the wave generation have been suggested so far, but all of them seem to have difficulties putting together all elements of the heating process.

The waves with high wavenumbers can occur throughout the corona due to a turbulent cascade starting from large MHD scales (e.g. Hollweg, 1986; Hollweg and Johnson, 1988; Isenberg, 1990; Li et al., 1999). However, this process rather produces waves with high perpendicular wavenumbers (e.g. Mongomery and Turner, 1981; Montgomery, 1982; Shebalin et al., 1983; Zank and Matthaeus, 1993; Ng and Bhattacharjee, 1996; Kinney and McWilliams, 1998; Matthaeus et al., 1998; Oughton et al., 1998; Nakayama, 1999; Milano et al., 2001), while generating high frequencies and parallel wavenumbers required for the cyclotron resonance remains a problem. A possible solution of this problem has been described by Cranmer and van Ballegooijen (2003), Vasquez et al. (2003).

Alternatively, the ion cyclotron waves can be excited by the plasma instability driven by currents associated with a global MHD mode (Markovskii, 2001). A similar idea was used by Viñas et al. (2000) to explain electron heating in the corona. But this channel of the wave generation does not operate for an arbitrarily small frequency of the global MHD mode compared to the proton cyclotron frequency. Therefore, it cannot be linked directly to the large-scale fluctuations, which are thought to be a possible source of the solar wind energy.

According to another scenario, the waves are directly launched at the coronal base by reconnection events (e.g. Axford and McKenzie, 1992; 1996; Marsch and Tu, 1997; Tu 
and Marsch, 1997; Ruzmaikin and Berger, 1998). The difficulty with this approach is that the ion cyclotron waves have to propagate through lower layers of the corona, where they can be heavily damped on the ions with a smaller charge-tomass ratio, before they reach upper layers and heat the ions with a larger charge-to-mass ratio (Cranmer, 2000; 2001). This argument favors the generation of the ion cyclotron waves throughout the corona rather than at the coronal base. $\mathrm{Tu}$ and Marsch (2001) argued, on the other hand, that the damping on minor ions is significant only if the wave energy density is relatively low. At greater densities, the damping can be negligible.

However, the energy densities used by Tu and Marsch (2001) to demonstrate the weak wave damping on the minor ions are large enough to trigger an additional damping mechanism. As shown by Markovskii and Hollweg (2002a), at these energy densities the waves experience additional damping due to the excitation of a plasma instability by the currents associated with the waves.

One more challenge to the direct-launching scenario was presented by Hollweg (2000). He found that the wave spectra used by Tu and Marsch (1997) are probably inconsistent with the observed spectrum of the density fluctuations in the corona. This contradiction can be avoided if all the waves propagate nearly along the coronal magnetic field, but this seems to be unlikely.

It should be emphasized that the above-mentioned difficulties do not definitively rule out any of the proposed mechanisms of the coronal heating. At the same time, we recently suggested a new approach involving plasma microinstabilities generated by large but intermittent electron heat flux in the corona. The aim of this paper is to develop these ideas further.

\section{Description of the heating model}

\subsection{Basic concepts}

The basic concepts of our model were introduced by Hollweg and Markovskii (2002) and Markovskii and Hollweg (2002b; 2003; 2004). Here we repeat some of them for completeness. The first element of our model is magnetic reconnection resulting in microflares at the coronal base. During the flares, a significant portion of the magnetic field energy goes to the thermal energy of electrons. The electrons are heated locally and when the hot particles become collisionless they escape from the heating site generating heat flux going up into the corona. This heat flux can be pictured as electron beams for simplicity, although in fact the distribution function is skewed rather than having a bump-on-tail structure. In a collisionless plasma, the electrons escaping from the site of the local heating can form a beam-like distribution function through velocity dispersion, at least at the initial stage of the evolution (e.g. Ledenev and Starygin, 2001).

At the next step in our model, the heat flux loses energy to protons. This is possible because the heat flux can ex- cite a plasma microinstability and the generated waves are cyclotron resonant with the protons. The heating of the solar wind by heat flux generated waves has been studied by Forslund (1970), Toichi (1971), and Coppi (Coppi, B., Collisionless collective modes including transverse ion heating, Unpublished talk presented at Solar Wind Nine, Nantucket Island, MA, 1998); for a review of the heat-flux instabilities see, e.g. Gary (1993). In our case, the instability has the same nature as a current-driven instability. A simple way to describe it is to consider two electron components: a hot and tenuous heat flux carrying beam and a relatively cold and dense core corresponding to the background electron population. The background temperature is about $10^{6} \mathrm{~K}$. The temperature of the hot component is greater by an order of magnitude. This is a reasonable estimate because if we assume that a 10 Gauss magnetic field reconnects and gives all of its energy to the electrons then, for typical densities of the order of $10^{8} \mathrm{~cm}^{-3}$ at the coronal base, the electrons can be heated up to $10^{8} \mathrm{~K}$. Therefore, there is more than enough energy residing in the magnetic field.

The components of the electron distribution move with respect to each other at the thermal speed of the hot component, and they also move with respect to the protons. This drift of the background electron population with respect to the protons can drive the instability as if there were a current, even though the total current is assumed to be zero. In particular, much like the current-driven instability, the heatflux instability generates electrostatic waves at lower values of the plasma $\beta$ and shear Alfvén waves at higher $\beta$ (e.g. Markovskii and Hollweg, 2002b; 2003). These waves can then interact with ions and heat them.

A different, but similar, approach was used by Feldman et al. (1993) and Voitenko and Goossens (2002). They suggested that MHD flows emanating from microflare reconnection sites can excite Alfvén waves. As has been shown by Voitenko and Goossens (2002), the excitation of kinetic Alfvén waves is due to a plasma microinstability. The unstable waves will heat the plasma in the vicinity of the coronal base. The propagation of the ion beams to higher altitudes has also been discussed by Feldman et al. (1997) and Voitenko and Goossens (2003). As pointed out by an anonymous referee, there is an important distinction between the waves generated by the ion and electron beams: they propagate in opposite directions. In this respect, the observational evidence of sunward/anti-sunward propagating density fluctuations in the corona would help us to distinguishing between these two mechanisms.

It is important that in our model the heating has to be intermittent. When the microflare occurs, the heat flux only exists in the flux tube connected to the flare site, because the particles do not move across the magnetic field. The next flare at the same site does not happen immediately. There is some waiting time between the flares. Therefore, the beams launched by the microflares have a finite extent along the background magnetic field and these localized beams are separated by periods of much smaller heat flux. Furthermore, it turns out that not only is the intermittency inevitable, but 
this mechanism can only work if the heat flux is intermittent. If the heat flux were constant and large enough to exceed the threshold of the instability, then it would carry much more energy than is needed to drive the solar wind (Markovskii and Hollweg, 2002b; 2003). This is impossible because this extra flux cannot be left unaccounted. We know that at $1 \mathrm{AU}$ the energy of the solar wind is mostly the kinetic energy of the protons and the heat flux is small. This means that by $1 \mathrm{AU}$, or actually much closer to the Sun, the heat flux has already given all of its energy to the solar wind.

Therefore, to avoid this contradiction, we use the argument of the intermittency. We assume that sporadically the heat flux is large enough to excite the instability, but because these sporadic bursts are rare, the total time-averaged heat flux is much smaller and it gives the correct energy to the solar wind. In fact, this is how we can estimate the degree of intermittency, i.e. the ratio of the time between the bursts to the burst time (see Sect. 3.2 below). We need to compare the energy budget of the solar wind and the threshold of the instability. At the coronal base the degree of intermittency turns out to be around several hundred (Markovskii and Hollweg, 2002b, 2003).

2.2 Plasma heating in the collision-dominated region of the solar wind

Markovskii and Hollweg (2004) have developed a simple method of calculating the heating during the heat flux burst. We will not repeat the details here. We only mention that it is sufficient to assume a marginal instability slightly above the threshold, so the calculation can be done in the quasilinear limit. The reason is that the characteristic time of the global, macroscopic, expansion of the solar wind is much greater than any characteristic time of all microscopic plasma processes except perhaps the collision time. For instance, the proton gyroperiod is about 6 orders of magnitude smaller than the expansion time at 1.5 solar radii. Therefore, even if the instability results only in slow heating on the microscopic scale, it can still be fast enough on the global scale to account for the observed heating of the solar wind.

The heating during the heat flux bursts contributes to the overall heating, which in this model is a summed effect of a burst and of what happens until the next burst. The solar wind behavior between the bursts is relatively simple. There is no energy input and the solar wind just cools down adiabatically because of its expansion. This kind of behavior is illustrated in Fig. 1. If we mark a plasma parcel and follow its motion with the solar wind, the temperature profile of this parcel will look like sawteeth, instead of a smooth line. By comparing the heating during the burst and the adiabatic cooling between the bursts, we can determine whether this mechanism maintains the temperature at the level suggested by the observations.

One of the problems associated with the intermittent heating is that the proton distribution function at the beginning of a heating cycle in Fig. 1 is not the same as it is at the end of the cycle. It gets distorted. There are several sources of the

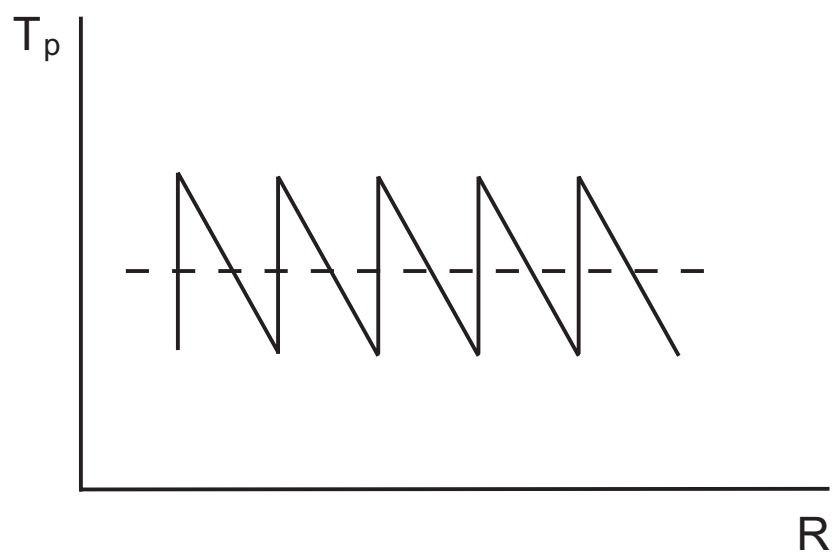

Fig. 1. Schematic of the proton temperature $T_{p}$ of a marked plasma parcel moving with the solar wind, which results from the intermittent heating, as a function of the heliocentric distance $R$. The temperature profile that would result from continuous heating is shown as a dashed line.

distortion. One of them is the heating itself. The wave particle interaction due to the heat flux instability operates only in a certain region of the particles phase space. As a result, the distribution deviates significantly from its initial structure during the burst. Another source of the distortion is the mirror force, which is different for particles with different velocities across the magnetic field. Therefore, in general, for every cycle we would need to calculate the heating for a different distribution. It turns out, however, that there is always a process that works against the distortion of the distribution function. This process can recycle the distribution and it will have qualitatively the same structure by the end of every cycle.

The situation is especially simple close to the Sun because, for reasonable parameters of the heat flux, the time between the bursts can be greater than the time of proton-proton collisions. As a result, the distortion from the heating is completely absorbed and the distribution is isotropized by the collisions by the time of the next burst. The distortion due to the mirror force also does not occur. This means that the variation of the macroscopic solar wind parameters can be calculated simply from fluid equations. Note that the particle diffusion within the burst is not affected by the collisions because the bursts are short enough.

The region close to the Sun, where the collision time is smaller than the interval between the heat flux bursts, is the most important one because the expansion here is the strongest and a heating mechanism should work most efficiently to overcome the adiabatic cooling. However, the heating continues at greater heliocentric distances and without the collisions we can again run into the problem of the distortion of the distribution function mentioned above. A detailed discussion of this problem is beyond the scope of the present paper. Here we only mention that the collisions can be replaced by a similar process. The basic idea is that the distribution function distorted by the mirror force between the 
bursts is unstable and excites waves that scatter particles and act against the distortion. This secondary instability cannot add any energy to the particles, in contrast with the primary heat-flux instability, because the secondary instability is generated by the distortion of the distribution and the distortion is due to a stationary magnetic field. Therefore, the heating will still be done only by the heat-flux instability during the burst. On the other hand, the scattering resulting from the secondary instability can play the role of collisional scattering and prevent the distribution from getting too far from the Maxwellian at the beginning of each burst. Note, however, that, in contrast with the collisional scattering, the scattering due to the secondary instability is anisotropic; therefore, complete isotropization is unlikely in this case.

Below, as in Markovskii and Hollweg (2004), we will focus on the collision-dominated region of the solar wind. A simple way to describe the global evolution of the solar wind in this region is to use the fluid equations with a heating function derived from the microscopic kinetic theory. In the fluid models, the heating function determines the amount of energy put into the solar wind by some unspecified mechanism at a given heliocentric distance and it plays the role of a source term in the MHD equation of energy.

To establish the analogy between the fluid models and our heating mechanism, we need to assume that the time between the heat flux bursts is much smaller than the solar wind expansion time. In this case, the variation of the macroscopic solar wind parameters will be determined by a time-averaged effect of many bursts and each burst will give only small variations of the solar wind parameters. Since the bursts are very short and the evolution of the solar wind between bursts is controlled by collisions, as discussed above, our mechanism will then give the same results as the fluid model if the heating function is the same.

The solar wind expansion time, which we define as the ratio of the scale of the density inhomogeneity to the solar wind speed, varies with the heliocentric distance. The minimum value of the expansion time is approximately $1000 \mathrm{~s}$ at 1.5 solar radii. Following Markovskii and Hollweg (2004), we assume that the time interval between the heat flux bursts $\Delta t$ is much smaller and equals to $100 \mathrm{~s}$.

Once this time is specified, we can estimate the maximum heliocentric distance $R_{\text {coll }}$ at which the distribution between the bursts can be recycled and isotropized by the collisions. We use the formula

$\tau_{p p}=0.29 \frac{\sqrt{m_{p}}(k T)^{3 / 2}}{n e^{4} \ln \Lambda}$

for the proton-proton collision time (e.g. Spitzer, 1962, Eqs. 5-26), where $\ln \Lambda$ is the Coulomb logarithm. Taking $T=10^{6} \mathrm{~K}$ and the density in the form

$n=3.2 \cdot 10^{8} r^{-15.6}+2.5 \cdot 10^{6} r^{-3.76} \mathrm{~cm}^{-3}$

derived from the observational data of Feldman et al. (1997), where $r$ is the heliocentric distance in solar radii, we obtain $R_{\text {coll }} \approx 1.3 R_{\text {sun }}$. Beyond $R_{\text {coll }}$, the quantity $\tau_{p p}$ is greater than $\Delta t$. The value of $R_{\text {coll }}$ is somewhat uncertain because $\Delta t$ is unknown, but apparently $R_{\text {coll }}$ cannot exceed $1.5 R_{\text {sun }}$, the distance at which the time between the bursts $\Delta t$ becomes comparable to the solar wind expansion time.

\subsection{Solution of the fluid equations}

To illustrate the heating and acceleration of the solar wind, we will use a numerical example similar to the one in Markovskii and Hollweg (2004). We start from the steadystate fluid equations of the mass, momentum, and energy conservation for the protons

$$
\begin{aligned}
n(R) V(R) A(R)= & \text { const, } \\
V(R) \frac{d V(R)}{d R}= & -\frac{1}{m_{p} n(R)} \frac{d\left[k n(R)\left(T_{p}(R)+T_{e}(R)\right)\right]}{d R} \\
& -\frac{G M_{\mathrm{sun}}}{R^{2}}, \\
V(R) \frac{d T_{p}(R)}{d R}= & -\frac{2}{3} \frac{T_{p}(R)}{A(R)} \frac{d[V(R) A(R)]}{d R} \\
& +\frac{2}{\tau_{e p}}\left(T_{e}(R)-T_{p}(R)\right)+\frac{2}{3 n k} Q(R),
\end{aligned}
$$

where $R$ is the heliocentric distance, $Q$ is the heating function, $A$ is the flow tube cross section, and

$$
\tau_{e p}=0.15 \frac{m_{p}\left(k T_{e}\right)^{3 / 2}}{\sqrt{m_{e}} n e^{4} \ln \Lambda}
$$

is the electron-proton collision time. The thermal coupling between the electrons and protons, described by the second term on the right-hand side of Eq. (5), is considerable within a distance of about $1.3 R_{\text {sun }}$, where $\tau_{e p}$ is less than the solar wind expansion time, and becomes insignificant at greater distances. Recall that the quantities entering into these equations are time-averaged ones as displayed in Fig. 1 by a dashed line. In general, the heat flux instability results in momentum addition as well as heating, because the particles acquire a bulk velocity along the magnetic field. The momentum addition can be incorporated into a fluid model (e.g. Esser et al., 1997). From a mathematical standpoint, it appears as a source or sink term in the momentum equation.

Our goal, however, is to check whether the heating rate associated with the heat flux in our model can provide observed parameters of the solar wind. The solar wind density $n(R)$ can be measured quite accurately; therefore, we assume that $n(R)$ is given by Eq. (2), derived from the observations, and consider $Q(R)$ an unknown function. We supplement the system of Eqs. (3)-(5) with the area expansion function $A(R)$ in the form

$A(R)=\frac{f_{\max }\left(R / R_{\text {sun }}\right)^{2}}{1+\left(f_{\max }-1\right)\left(1+\left(\left(R / R_{\text {sun }}\right)-1\right)^{b}\right)^{-c}}$.

Here $f_{\max }$ is the overall expansion factor of the flow tube, which we set equal to 5 . The constants $b$ and $c$ determine how the overall expansion is distributed as a function of the 
heliocentric distance. We put $b=2$ and $c=7$. This assures that, firstly, the temperature maximum of the solution obtained below is achieved beyond 1.5 solar radii and, secondly, the faster than radial expansion contributes a factor of 2.7 to the flow tube cross section from 1 to 1.5 solar radii, which will result in a solar wind speed of $100 \mathrm{~km} / \mathrm{s}$ at $1.5 R_{\text {sun }}$ for the boundary conditions described below. According to Eq. (7), the greatest rate of the superradial expansion occurs near $1.5 R_{\text {sun }}$ and the expansion is essentially radial beyond $2.5 R_{\text {sun }}$.

We then solve Eqs. (3)-(5) with the boundary condition $T_{p}\left(1.5 R_{\text {sun }}\right)=1.5 \cdot 10^{6} \mathrm{~K}$, which is consistent with the observations reported by Esser et al. (1999). The constant in Eq. (3) is equal to the proton flux density at $1 \mathrm{AU}$ $3 \cdot 10^{8} \mathrm{~cm}^{-2} \mathrm{~s}^{-1}$. For simplicity, we do not attempt to describe the electrons self-consistently. Instead, we set the electron temperature to be almost constant and never greater than the proton temperature in agreement with the observational data in the corona (Wilhelm et al., 1998). We take into account the electron-proton thermal coupling by assuming that the electron and proton temperatures are exactly equal for $R<1.3 R_{\text {sun }}$ with $T_{e}$ gradually leveling off to a constant value of $8 \cdot 10^{5} \mathrm{~K}$ at $R>1.3 R_{\text {sun }}$. The numerical solutions for $T_{p}(R)$ and $T_{e}(R)$ are displayed in Fig. 2. These temperature profiles are close to the ones obtained by Markovskii and Hollweg (2004) in the approximation of an exactly constant electron temperature $T_{e}=7.5 \cdot 10^{5} \mathrm{~K}$ neglecting the electronproton thermal coupling in Eq. (5). The reason is that in the region $R<1.3 R_{\text {sun }}$, where the coupling could affect the energy balance, the proton and electron temperatures calculated from Eqs. (3)-(5) turn out to be close to each other even if the coupling is neglected. However, for completeness, we have used a more accurate model here. We do not extend this solution beyond 1.5 solar radii because the isotropic fluid equations are not applicable to our model at greater distances (see the discussion in the end of Sect. 2.2). At the same time, within 1.5 solar radii these solutions give the temperature values close to the observed ones; and the heating rate, determined by Eq. (5), is comparable to the one used, for instance, by Esser et al. (1997) in their fluid model of the solar wind.

\section{Parameters of the heat flux}

Now our goal is to estimate the heat flux required to produce the solar wind parameters shown described above and then check if it is consistent with the actual radial evolution of the electron beam carrying the heat flux.

\subsection{Heat flux derived from the heating rate}

The heat flux is controlled by three parameters of the electron beam, its bulk speed, temperature, and density (see Eq. 13 below). For an illustration, we postulate that the beam electrons are heated up to the temperature $T_{b}=8 \cdot 10^{6} \mathrm{~K}$ at the coronal base and the beam is launched at the bulk speed equal to its thermal speed $V_{T b}=\sqrt{2 k T_{b} / m_{e}} \approx 15600 \mathrm{~km} / \mathrm{s}$. Follow-

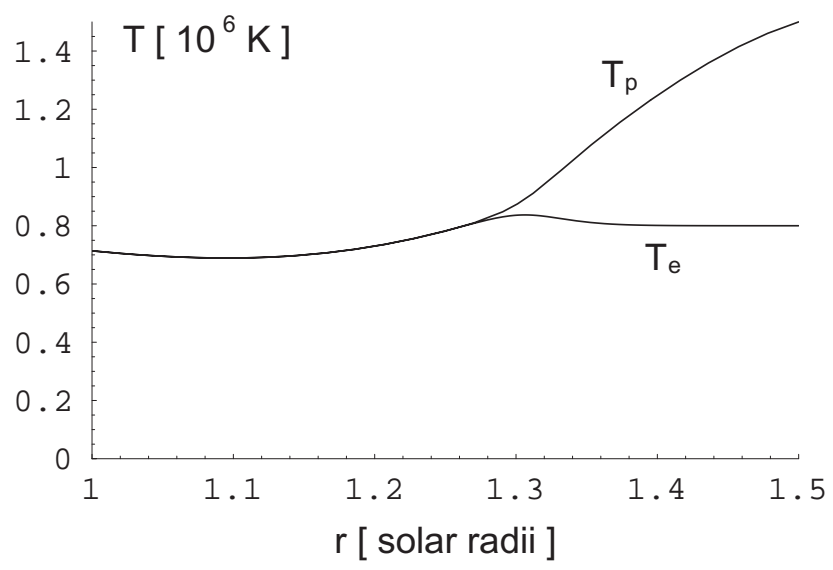

Fig. 2. Numerical solution of the fluid Eqs. (3)-(5) for the solar wind electron and proton temperatures as functions of the heliocentric distance.

ing Markovskii and Hollweg (2004), we will also assume that the beam's speed and temperature remain constant as it propagates to higher altitudes. Then, the beam density will be the only varying parameter. As we will see below, in Sect. 3.2, this is a reasonable approximation at least in our region of interest, within $1.5 R_{\text {sun }}$.

As shown by Markovskii and Hollweg (2004), the heat flux instability needed to provide the heating rate resulting from Eqs. (3)-(5) has to be in a marginal state. Therefore, to derive the radial evolution of the heat flux from the heating rate, we can simply determine the threshold beam density required to excite the instability for the beam speed and temperature specified in the previous paragraph. In the low- $\beta$ region of the corona close to the Sun, the dominant mode of the heat-flux instability is the electrostatic one. To calculate the threshold beam density, we will use the usual linear electrostatic dispersion relation in the frame moving at the bulk speed of the protons:

$1+\varepsilon_{p}(\omega)+\varepsilon_{c}\left(\omega-k_{\|} u_{c}\right)+\varepsilon_{b}\left(\omega-k_{\|} u_{b}\right)=0$,

where the subscripts $p, c$, and $b$ denote the protons and the background (core) and heat flux carrying (beam) electron components, respectively. The relative bulk velocities $u_{c}$ and $u_{b}$ of the electron components with respect to the protons satisfy the zero current condition

$n_{c} u_{c}+n_{b} u_{b}=0$

where $n_{c}$ and $n_{b}$ are the number densities of the electron components. The real part of the angular frequency is $\omega$ and the subscript $\|$ denotes the direction parallel to the background magnetic field $\boldsymbol{B}_{0}=\left(0,0, B_{z 0}\right)$. The particle distributions of the protons and the electron core and beam are assumed to have the Maxwellian form. The explicit expressions for $\varepsilon$ 's can be found, e.g. in Markovskii and Hollweg (2004).

When the beam speed is constant, the threshold of the heat-flux instability depends most strongly on the temperature ratio of the protons and the core electrons. The threshold 


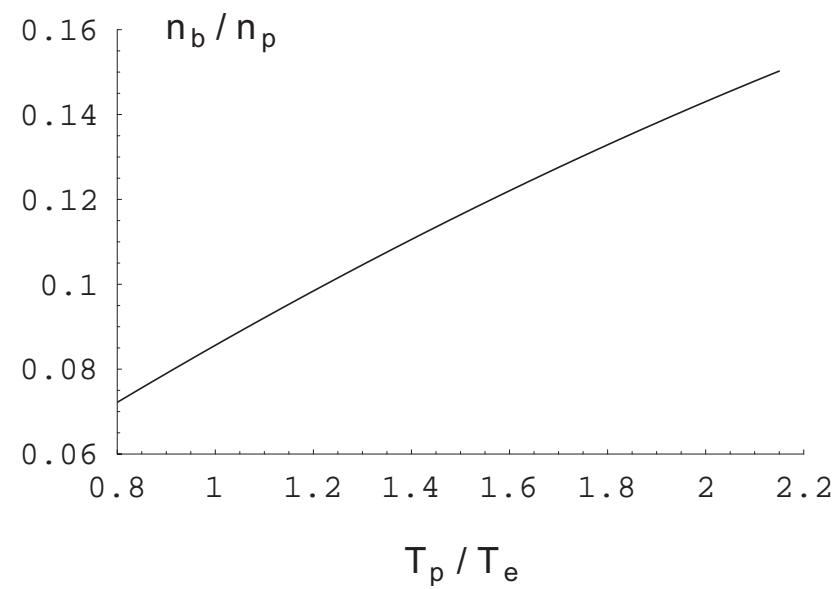

Fig. 3. Threshold relative beam density as a function of the protonto-electron temperature ratio.

relative beam density calculated from Eq. (8) as a function of the temperature ratio is plotted in Fig. 3. We then used the proton and electron temperatures calculated from the fluid equations and shown in Fig. 2 to represent the threshold beam density as a function of the heliocentric distance. The result is displayed as the top curve in Fig. 4. The electrostatic dispersion depends on the Alfvén speed very weakly as long as $V_{A} / c \ll 1$. Therefore, we assumed the same Alfvén speed $V_{A}=3000 \mathrm{~km} / \mathrm{s}$ in the entire region from 1 to 1.5 solar radii.

\subsection{Heat flux derived from the beam evolution}

The actual radial evolution of the heat-flux carrying beam is determined by three processes. Firstly, because the beam has a finite spatial extent, it expands along the magnetic field due to the velocity dispersion of the beam particles. This expansion by itself is not accompanied by the beam energy variation, since the particles are hot enough and they interact neither among themselves nor with the background particles via collisions. Secondly, the beam loses its energy to the protons via the wave-particle interactions resulting from heat flux instability and the interaction with the electromagnetic field responsible for the zero-current condition (Eq. 9). Thirdly, the beam loses its energy due to the global forces in the corona. The most important forces are the mirror force in the inhomogeneous magnetic field and the electric field created by the charge separation of the protons and the background electrons, while the effect of gravitational force acting on the beam electrons is negligible. This latter energy loss is undesirable from the standpoint of our model, because the beam loses the energy without heating the protons.

The full description of beam evolution is a complicated problem, which is beyond the scope of the present paper. We will defer the second process associated with the heatflux instability to a future study. Here we will show that the beam's thermal expansion and the action of the global forces are consistent with our heat-flux instability mechanism.

Let us first estimate the variation of the beam speed as it propagates away from the Sun. To model the beam's bulk

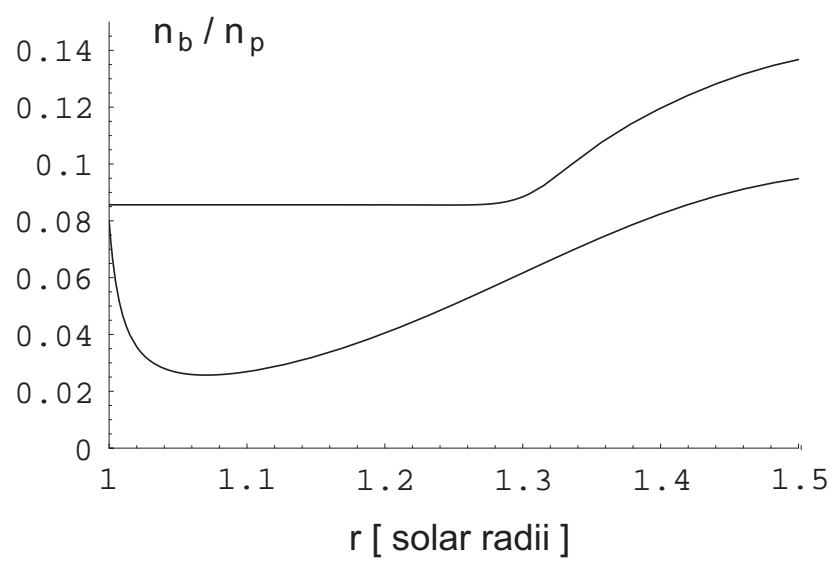

Fig. 4. Relative beam density as a function of the heliocentric distance calculated from the heating rate (top curve) and from the beam expansion (bottom curve).

motion, we take a quasi-particle that represents the most populated region of the beam distribution. The initial velocity $v_{\| 0}$ of the quasi-particle along the magnetic field at the coronal base is equal to the beam bulk speed and the initial perpendicular velocity $v_{\perp 0}$ is equal to the beam thermal speed. We assume that the beam is launched at the coronal base with the temperature $T_{b}=8 \cdot 10^{6} \mathrm{~K}$ and the bulk speed $V_{T b}=\sqrt{2 k T_{b} / m_{e}}$, and the radial direction coincides with the direction of the magnetic field. Thus, both $v_{\| 0}$ and $v_{\perp 0}$ are equal to $V_{T b}$. The acceleration of the quasi-particle in the radial direction is determined by the mirror force and the charge separation electric field:

$\frac{d v_{\|}}{d t}=\frac{v_{\perp}}{2} \frac{d(\ln A)}{d R}+\frac{k}{m_{e} n} \frac{d\left(n T_{e}\right)}{d R}$,

where the last term on the right-hand side is the electron pressure gradient with a constant electron temperature, which is responsible for the generation of the electric field, $A$ and $n$ are given by Eqs. (7) and (2), respectively, and $T_{e}$ is displayed in Fig. 2. We then solve Eq. (10) using the equality $d R / d t=v_{\|}$, the relation $A(R) \sim B^{-1}(R)$, and the conservation of the magnetic moment, which implies $v_{\perp}^{2}(R) \sim B(R)$.

The resulting solution for $v_{\| 0}$ with the boundary conditions $v_{\| 0}=v_{\perp 0}=15600 \mathrm{~km} / \mathrm{s}$ is shown in Fig. 5. As we can see, the velocity is almost constant (in agreement with our assumption in the previous section) with a slight acceleration due to the mirror force. At the same time, the total kinetic energy of the quasi-particle, modeling the total energy of the beam, is decreasing (Fig. 6) at the expense of the perpendicular energy because the mirror force does not change the particle's total energy and the electric field extracts the parallel energy. However, the energy variation is not significant, the quasi-particle loses about $1 / 4$ of its initial energy, which is again consistent with our assumption in Sect. 3.1. We do not extend the solution beyond $1.5 R_{\text {sun }}$ because the approximation of an almost constant electron core temperature eventually becomes invalid at greater distances. 


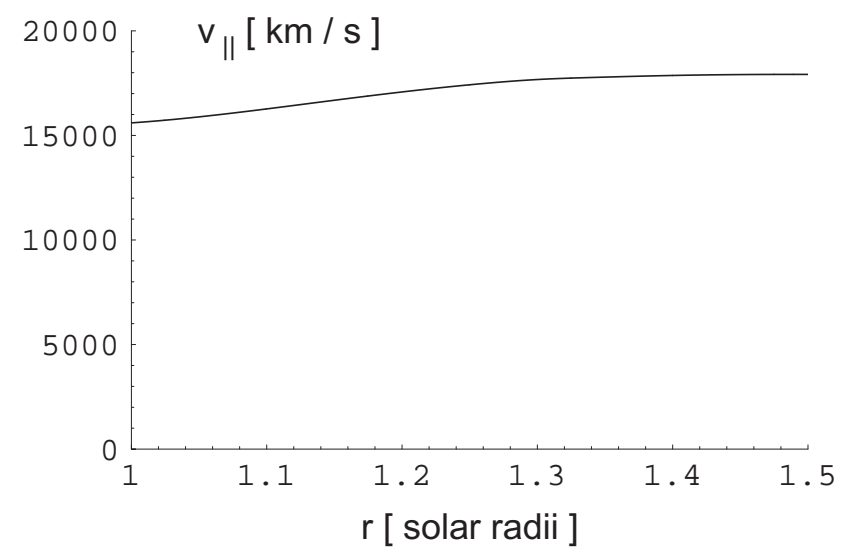

Fig. 5. Calculated speed of the quasi-particle modeling the bulk speed of the beam as a function of the heliocentric distance.

Note that the total beam energy, as well as the actual beam distribution function, has only a minor effect on the excitation of the heat flux instability. The reason is that the beam particles do not interact with the waves directly. The only role of the beam is to produce the backstreaming of the background electrons through the zero current condition (Eq. 9) and it is the background electrons that drive the instability. Therefore, the excitation of the instability is controlled mainly by the beam bulk speed and density. It is important to emphasize that the backstreaming of the electron core is not affected by the electron-proton collisions, because the backstreaming takes place only when the finite-extent beam passes through a given region of the background plasma within a period of time much smaller than the electron-proton collision time.

To calculate the radial evolution of the beam density, we now need to determine the parameters of the heat flux at the coronal base. As discussed above, the heat flux has to be intermittent. The degree of intermittency is derived from the energy budget of the solar wind in the following way (Markovskii and Hollweg, 2002b; 2003). At 1 AU the energy of the solar wind is mostly in the form of the proton kinetic energy. Taking the number density at $1 \mathrm{AU} n_{E}=4 \mathrm{~cm}^{-3}$ and the solar wind speed $V_{E}=750 \mathrm{~km} / \mathrm{s}$, we find the energy flux per unit area per unit time $q_{E}=1.4 \mathrm{erg} \cdot \mathrm{cm}^{-2} \cdot \mathrm{s}^{-1}$. Mapping this flux back to 1 solar radius and assuming that the coronal holes occupy $20 \%$ of the solar surface, we obtain the energy flux at the coronal base $q=3.3 \cdot 10^{5} \mathrm{erg} \cdot \mathrm{cm}^{-2} \cdot \mathrm{s}^{-1}$.

The flux $q$ consists mostly of two parts: the heat flux $q_{T}$ carried by the electrons and a negative gravitational flux $q_{G}$

$q=q_{T}+q_{G}$,

where

$q_{G}=-m_{p} n_{p} V \frac{G M_{\text {sun }}}{R_{\text {sun }}}$,

$G$ is the gravitational constant, $M_{\text {sun }}$ is the solar mass, and

$q_{T}=\frac{\delta t}{\Delta t} \frac{m_{e}}{2} \sum_{l} n_{l} u_{l}\left(\frac{5 k T_{l}}{m_{e}}+u_{l}^{2}\right)$.

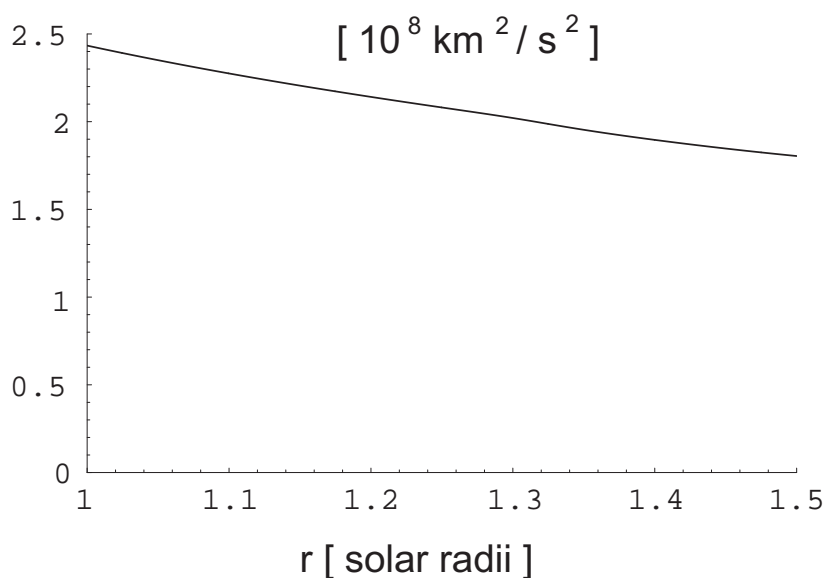

Fig. 6. Total energy of the quasi-particle per unit mass as a function of the heliocentric distance.

Here the sum is over the beam and core (background) components of the electrons population, the heat flux is calculated for a Maxwellian distribution of each component, $\delta t$ is the duration of the heat flux burst, $\Delta t$ is the time interval between the heat flux bursts, and the ratio $\Delta t / \delta t$ is the degree of intermittency. Strictly speaking, Eq. (11) is incomplete because not all of the beam energy goes to the protons. As discussed above, some of the energy is needed to get the beam itself out of the potential well associated with the charge-separation electric field. However, given the qualitative nature of our calculations, Eq. (11) is a reasonable approximation.

Substituting the parameters at the coronal base $T_{b}=10 T_{c}$, $u_{b}=V_{T b}, T_{b}=8 \cdot 10^{6} \mathrm{~K}$, and $n_{b} / n_{p}=0.08$, using Eqs. (7) and (13), and deriving the solar wind speed at the base $V=2.1 \mathrm{~km} / \mathrm{s}$ from the mass conservation law, we calculate the degree of intermittency at the coronal base $\Delta t /(\delta t)_{0}=260$. From here the burst duration $(\delta t)_{0}=\Delta t / 260=0.38 \mathrm{~s}$.

The only difference at greater heliocentric distances is that the heat flux becomes less intermittent. The duration of the heating cycle $\Delta t$ remains the same, but the duration of the heat flux burst $\delta t$ increases. The reason is that, as discussed above, the heat flux carrying electron beam has a finite spatial extent and it is expanding along the background magnetic field as it moves away from the Sun. Since the beam electrons are collisionless, the beam expands roughly at the root mean square velocity of the beam particles in the direction of the magnetic field.

To estimate the beam density at a given distance, we will assume that the front and rear edges of the beam, moving at the velocities $u_{f 0}=u_{b 0}+0.5 \sqrt{{<v_{z}^{2}>_{b}}_{0}} \approx 21000 \mathrm{~km} / \mathrm{s}$ and $u_{r 0}=u_{b 0}-0.5 \sqrt{<v_{z}^{2}>_{b}} \approx 10000 \mathrm{~km} / \mathrm{s}$, respectively, start at the coronal base with the time delay $(\delta t)_{0}$. Here $u_{b}=V_{T b} \approx 15600 \mathrm{~km} / \mathrm{s}$ and

$$
<v_{z}^{2}>_{b}=\sqrt{\frac{m_{e}}{2 \pi k T_{b}}} \int_{-\infty}^{+\infty} v_{z}^{2} \exp \left(-\frac{m_{e} v_{z}^{2}}{2 k T_{b}}\right) d v_{z} .
$$


We then model the beam's rear and front edges again with two quasi-particles whose initial perpendicular velocity is equal to $V_{T b}$ and the parallel velocities are equal to $u_{r}$ and $u_{f 0}$, respectively.

The beam density is inversely proportional to the increasing distance between the front and rear quasi-particles and the increasing flux tube cross-section $A(R)$. From here we obtain

$$
\frac{n_{b}}{n_{p}}=\frac{n_{b}\left(R_{\text {sun }}\right)}{n_{p}\left(R_{\text {sun }}\right)} \frac{n_{p}\left(R_{\text {sun }}\right)}{n_{p}(R)} A^{-1}(R) \frac{(\delta t)_{0} u_{r 0}}{R-R_{r}},
$$

where we set $R$ equal to the position of the beam's front edge $R_{f}$ and $R_{f}$ is related to the position of the rear edge $R_{r}$ by the equation

$$
\int_{R_{\text {sun }}}^{R_{f}} \frac{d s}{v_{f}(s)}-(\delta t)_{0}=\int_{R_{\text {sun }}}^{R_{r}} \frac{d s}{v_{r}(s)} .
$$

Here $v_{f}(R)$ and $v_{r}(R)$ are the solutions of Eq. (10) describing the front and rear quasi-particles. The quantity $n_{b} / n_{p}$ is plotted in Fig. 4 (bottom curve) as a function of the heliocentric distance $R$.

As we see, the beam's relative density derived from its evolution stays approximately constant compared to the absolute density that drops by more than two orders of magnitude in this region. This behavior is similar to that of the relative density derived from the heating rate, which is almost constant too. Also, both curves in Fig. 6 follow the same trend, which is a reasonably good agreement in the framework of our qualitative model.

\section{Summary}

In summary, the microflares at the coronal base heat electrons and generate intermittent heat flux going up into the corona. The heat flux excites an ion cyclotron instability, which results in ion heating. The intermittent heat flux produced by the microflares was described as electron beams, characterized by their bulk speed, density, and thermal energy. We have calculated the beam evolution under the action of the mirror force and the charge separation electric field. The mirror force is due to the inhomogeneous magnetic field and the electric field is generated by the pressure gradient of the background electron population. We modeled the beam with quasi-particles representing the most populated region of its distribution.

We have shown that the beam bulk speed stays almost constant and the total energy decreases slightly in the collisiondominated region of the corona close the Sun. A large energy loss due to the charge-separation electric field would be undesirable from the standpoint of our model, because the beam loses energy without heating the protons. At the same time, the total beam energy, as well as the actual beam distribution function, has only a minor effect on the excitation of the heat flux instability. The reason is that beam particles do not interact with the waves directly. The only role of the beam is to produce the backstreaming of the background electrons through the zero-current condition and it is the background electrons that drive the instability. Therefore, the excitation of the instability is controlled by the beam bulk speed and density.

Since the beam speed and energy do not change much, the radial evolution of the heat flux is determined by the expansion of the finite-extent beam along the magnetic field roughly at the root mean square velocity of the beam particles. This expansion by itself is not accompanied by the beam energy variation, since the particles are hot enough and they interact neither among themselves nor with the background particles via collisions. The only varying quantity is then the beam density. We have demonstrated that the beam density variation is not inconsistent with the heating rate required to produce the observed solar wind parameters.

Finally, in this paper, we have not taken into account the effect of the microinstability on the evolution of the electron distribution function. The microinstability will introduce additional decrease of the energy of the heat-flux carrying beam. However, the energy will be lost to the protons, which is what we want to achieve with this model. As the threshold of the heat-flux instability gradually decreases with the heliocentric distance, all of the energy is eventually extracted from the beam, and the core and beam will slow down to the speeds observed in situ in the solar wind. However, a proper kinetic calculation of this process still remains to be done.

Acknowledgements. This work is supported by NSF under grant ATM-0211402 and by NASA's Sun-Earth Connection Theory program under grant NAG5-11797, Solar and Heliospheric Physics SR\&T program under grant NAG5-10988, and Living With a Star program under grant NAG5-10835 to the University of New Hampshire.

Edited by: J. Büchner

Reviewed by: two referees

\section{References}

Antonucci, E., Dodero, M. A., and Giordano, S.: Fast solar wind velocity in a polar coronal hole during solar minimum, Sol. Phys., 197, 115-134, 2000.

Axford, W. I. and McKenzie, J. F.: The origin of high speed solar wind streams, in Solar Wind Seven, edited by Marsch, E. and Schwenn, R., Pergamon, Tarrytown, N. Y., 1-5, 1992.

Axford, W. I. and McKenzie, J. F.: The acceleration of the solar wind, in Solar Wind Eight, edited by Winterhalter, D., McComas, D., Phillips, J., and Murphy, N., Am. Inst. Phys., Woodbury, N. Y., 72-75, 1996.

Cranmer, S. R.: Ion cyclotron wave dissipation in the solar corona: The summed effect of more than 2000 ion species, Astrophys. J., 532, 1197-1208, 2000.

Cranmer, S. R.: Ion cyclotron diffusion of velocity distributions in the extended solar corona, J. Geophys. Res., 106, 24 937-24 954, 2001. 
Cranmer, S. R. and van Ballegooijen, A. A.: Alfvénic turbulence in the extended solar corona: Kinetic effects and proton heating, Astrophys. J., 594, 573-591, 2003.

Dodero, M. A., Antonucci, E., Giordano, S., and Martin, R.: Solar wind velocity and anisotropic coronal kinetic temperature measured with the O VI doublet ratio, Sol. Phys., 183, 77-90, 1998.

Esser, R., Habbal, S. R., Coles, W. A., and Hollweg, J. V.: Hot protons in the inner corona and their effect on the flow properties of the solar wind, J. Geophys. Res., 102, 7063-7074, 1997.

Esser, R., Fineschi, S., Dobrzycka, D., Habbal, S. R., Edgar, R. J., Raymond, J. C., Kohl, J. L., and Guhathakurta, M.: Plasma properties in coronal holes derived from measurements of minor ion spectral lines and polarized white light intensity, Astrophys. J., 510, L63-L67, 1999.

Feldman, W. C.,Gosling, T. J., McComas, D. J., and Phillips, J. L.: Evidence for ion jets in the high-speed solar wind, J. Geophys. Res., 98, 5593-5605, 1993.

Feldman, W. C., Habbal, S. R., Hoogeveen, G., and Wang, Y.-M.: Experimental constraints on pulsed and steady state model of the solar wind near the Sun, J. Geophys. Res., 102, 26905-26918, 1997.

Forslund, D. W.: Instabilities associated with heat conduction in the solar wind and their consequences, J. Geophys. Res., 75, 17-28, 1970.

Gary, S. P.: Theory of Space Plasma Microinstabilities, Cambridge Univ. Press, Cambridge, UK, 156, 1993.

Hollweg, J. V.: Transition region, corona, and solar wind in coronal holes, J. Geophys. Res., 91, 4111-4125, 1986.

Hollweg, J. V.: Compressibility of ion-cyclotron and whistler waves: Can radio measurements detect high-frequency waves of solar origin in the corona?, J. Geophys. Res., 105, 7573-7582, 2000.

Hollweg, J. V. and Isenberg, P. A.: Generation of the fast solar wind: A review with emphasis on the resonant cyclotron interaction, J. Geophys. Res., 107, A7, SSH12-1-SSH12-37, doi:10.1029/2001JA000270, 2002.

Hollweg, J. V. and Johnson, W.: Transition region, corona, and solar wind in coronal holes: Some two-fluid models, J. Geophys. Res., 93, 9547-9554, 1988.

Hollweg, J. V. and Markovskii, S. A.: Cyclotron resonances of ions with obliquely propagating waves in coronal holes and the fast solar wind, J. Geophys. Res., 107, 6, SSH1-1-SSH1-7, doi:10.1029/2001JA000205, 2002.

Isenberg, P. A.: Investigations of a turbulent-driven solar wind model, J. Geophys. Res., 95, 6437-6442, 1990.

Kinney, R. M. and McWilliams, J. C.: Turbulent cascades in anisotropic magnetohydrodynamics, Phys. Rev. E, 57, 71117121, 1998.

Kohl, J. L., Noci, G., and Antonucci, E. et al.: UVCS/SOHO empirical determinations of anisotropic velocity distributions in the solar corona, Astrophys. J., 501, L127-L131, 1998.

Kohl, J. L., Esser, R.; Cranmer, S. R. et al.: EUV spectral line profiles in polar coronal holes from 1.3 to $3.0 \mathrm{R}$, Astrophys. J., 510, L59-L62, 1999.

Ledenev, V. G. and Starygin, A. P.: Heat transport from the region of local electron heating in a plasma, Plasma Phys. Rep., 27, 652-658, 2001

Li, X., Habbal, S. R., Hollweg, J. V., and Esser, R.: Heating and cooling of protons by turbulence-driven ion cyclotron waves in the fast solar wind, J. Geophys. Res., 104, 2521-2536, 1999.

Markovskii, S. A.: Generation of ion cyclotron waves in coronal holes by a global resonant magnetohydrodynamic mode, Astro- phys. J., 557, 337-342, 2001.

Markovskii, S. A. and Hollweg, J. V.: Parametric cross-field current instability in solar coronal holes, J. Geophys. Res., 107, 10, SSH21-1-SSH21-11, 1329, doi:10.1029/2001JA009140, 2002a.

Markovskii, S. A. and Hollweg, J. V.: Electron heat flux instabilities in coronal holes: Implications for ion heating, Geophys. Res. Lett., 29, 17, 21-1-24-4, 1843, doi:10.1029/2002GL015189, 2002b.

Markovskii, S. A. and Hollweg, J. V.: Ion heating due to plasma microinstabilities in coronal holes and the fast solar wind, in: Solar Wind Ten, edited by: Velli, M., Bruno, R., and Malara, F., AIP Conf. Proc. No. 679, 307-310, 2003.

Markovskii, S. A. and Hollweg, J. V.: Intermittent heating of the solar corona by heat flux-generated ion cyclotron waves Astrophys. J., 609, 1112-1122, 2004.

Marsch, E. and Tu, C. Y.: The effects of high-frequency Alfvén waves on coronal heating and solar wind acceleration, Astron. Astrophys., 319, L17-L20, 1997.

Matthaeus, W. H., Oughton, S., Ghosh, S., and Hossain, M.: Scaling of anisotropy in hydromagnetic turbulence, Phys. Rev. Lett., 81, 2056-2059, 1998.

Milano, L. J., Matthaeus, W. H., Dmitruk, P., and Montgomery, D. C.: Local anisotropy in incompressible magnetohydrodynamic turbulence, Phys. Plasmas, 8, 2673-2681, 2001.

Montgomery, D. C.: Major disruptions, inverse cascades, and the Strauss equations, Phys. Scr., T2:1, 83-88, 1982.

Montgomery, D. C. and Turner, L.: Anisotropic magnetohydrodynamic turbulence in a strong external magnetic field, Phys. Fluids, 24, 825-831, 1981.

Nakayama, K.: Statistical theory of anisotropic magnetohydrodynamic turbulence: An approach to strong shear Alfvén turbulence by direct-interaction approximation, Astrophys. J., 523, 315-327, 1999.

$\mathrm{Ng}, \mathrm{C}$. S. and Bhattacharjee, A.: Interaction of shear-Alfvén packets: Implication for weak magnetohydrodynamic turbulence in astrophysical plasmas, Astrophys. J., 465, 845-854, 1996.

Oughton, S., Matthaeus, W. H., and Ghosh, S.: Scaling of spectral anisotropy with magnetic field strength in decaying magnetohydrodynamic turbulence, Phys. Plasmas, 5, 4235-4242, 1998.

Ruzmaikin, A. and Berger, M. A.: On a source of Alfvén waves heating the solar corona, Astron. Astrophys., 337, L9-L12, 1998.

Shebalin, J. V., W. H. Matthaeus, and D. Montgomery: Anisotropy in MHD turbulence due to a mean magnetic field, J. Plasma Phys., 29, 525-547, 1983.

Spitzer, L.: Physics of fully ionized gases, New York, Interscience Publishers, 1962.

Toichi, T.: Thermal properties of the solar wind plasma, Sol. Phys., 18, 150-164, 1971.

Tu, C.-Y. and Marsch, E.: Two-fluid model for heating of the solar corona and acceleration of the solar wind by high-frequency Alfvén waves, Sol. Phys., 171, 363-391, 1997.

Tu, C.-Y. and Marsch, E.: Wave dissipation by ion cyclotron resonance in the solar corona, Astron. Astrophys., 368, 1071-1076, 2001.

Vasquez, B. J., Markovskii, S. A. and Hollweg, J. V.: Nonlinear Alfven waves, II, The influence of wave advection and finite wavelength effects, J. Geophys. Res., 109, A5, A05104, doi:10.1029/2003JA010106, 2004.

Viñas, A. F., Wong, H. K., and Klimas, A. J.: Generation of electron suprathermal tails in the upper solar atmosphere: Implications for coronal heating, Astrophys. J., 528, 509-523, 2000. 
Voitenko, Yu. and Goossens, M.: Excitation of high-frequency Alfvén waves by plasma outflows from coronal reconnection events, Sol. Phys., 206, 285-313, 2002.

Voitenko, Yu. and Goossens, M.: Kinetic excitation mechanisms for ion-cyclotron kinetic Alfvén waves in Sun-Earth connection, Space Sci. Rev., 107, 387-401, 2003.
Wilhelm, K., Marsch, E., Dwivedi, B. N., Hassler, D. M., Lemaire, P., Gabriel, A. H., and Huber, M. C. E.: The solar corona above polar coronal holes as seen by SUMER on SOHO, Astrophys. J., 500, 1023-1038, 1998.

Zank, G. P. and Matthaeus, W. H.: Nearly incompressible fluids, II, Magnetohydrodynamics, turbulence, and waves, Phys. Fluids, 5 257-273, 1993. 